\title{
ESTUDIO CORRELACIONAL ENTRE LAS PRUEBAS DE BACHILLERATO EN LA EDUCACIÓN MEDIA Y EL RENDIMIENTO EN EDUCACIÓN DIVERSIFICADA
}

\author{
Tania Elena Moreira Mora
}

Recibido 19-III-2002

- Aceptado 9-IV-2002

\section{Antecedentes}

En 1986 se realizó un diagnóstico curricular en estudiantes del Tercer Ciclo de la Educación General Básica y, a raíz de sus resultados, se decidió restablecer las pruebas de Bachillerato en Educación Media a partir de 1988. Para tal propósito se estableció un convenio entre el Ministerio de Educación Pública y el Instituto de Investigación para el Mejoramiento de la Educación Costarricense (IIMEC); para que las pruebas fueran elaboradas por docentes de secundaria, destacados en esta institución como investigadores, con la participación complementaria de los asesores nacionales de las asignaturas evaluadas.

El 15 de abril de 1997 se creó el Centro Nacional de Evaluación para la Educación (CENE-EDU) como órgano desconcentrado del Consejo Superior de Educación, según decreto ejecutivo № 26014-MEP. Este órgano fue el responsable de organizar, desarrollar y ejecutar las acciones de evaluación del sistema educativo formal, en sus diversas etapas y modalidades, con carácter nacional y para propósitos diagnósticos, formativos y sumativos (Art. 2). Entre otras funciones, asumió la elaboración, administración y calificación de las pruebas nacionales de acreditación y la evaluación de diversos proyectos y programas educativos. Este centro funcionó hasta junio de 1998. 
A partir de ese año, la División de Control de Calidad y Macroevaluación del Sistema Educativo ha asumido la responsabilidad de controlar la calidad del sistema educativo y acreditar a los estudiantes que han concluido sus estudios en Segundo y Tercer Ciclo de la Educación General Básica y en la Educación Diversificada.

En el Informe nacional de resultados de Bachillerato $1998^{1}$ se indicó que el fin primordial del Bachillerato en Educación Media es acreditar al estudiante egresado de la Educación Diversificada, con la aplicación de pruebas estandarizadas de las asignaturas consideradas básicas.

De acuerdo con los objetivos del bachillerato, hasta el año 1994, las pruebas se diseñaron con referencia a normas; con el fin de interpretar los resultados según la posición del estudiante dentro del grupo, con base en una norma determinada, en este caso, una escala de calificación de 0 a 100 , cuyo mínimo para aprobar era de 65 , sumando la nota de presentación (40\%) con la calificación del examen (60\%).

Para la construcción de las pruebas, los asesores nacionales en macroevaluación de cada una de las asignaturas, procedieron a elaborar un listado de contenidos conforme con los programas de estudio vigentes.

Desde 1995 hasta 1998 las pruebas se diseñaron con referencia a objetivos para: acreditar el nivel de logro de los objetivos del currículum nacional básico alcanzado por los estudiantes, recopilar información sobre las áreas prioritarias y realimentar al sistema educativo.

Las pruebas con referencia a objetivos fue un abordaje intermedio entre normas y criterios, pues en la construcción e interpretación de los resultados se midió el nivel de logro de un conjunto de aprendizajes establecidos en el currículum nacional básico. La interpretación se basó en el número o porcentaje de ítemes contestados correctamente para el conjunto de objetivos medidos o por el número de puntos acumulados.
En consonancia con este abordaje, los evaluadores encargados de la construcción y montaje de las pruebas, en coordinación con los asesores nacionales respectivos, elaboraron los Temarios unificados para las pruebas nacionales de acreditación para bachillerato. En estos se describieron los contenidos y objetivos temáticos. Estos últimos se plantearon de acuerdo con los diversos niveles cognitivos de la taxonomía de Benjamín Bloom.

De acuerdo con una directriz emanada por el Ministro de Educación Pública (Oficio DM 3292-98), en 1999 las pruebas se diseñaron nuevamente con referencia a normas y según el Reglamento de Evaluación de los aprendizajes ${ }^{2}$, en el artículo 126, las pruebas serán comprensivas de la materia desarrollada durante la Educación Diversificada y versarán sobre los contenidos programáticos correspondientes a las disciplinas de: Español, Estudios Sociales y Cívica, Ciencias, Matemática e Idioma extranjero.

Por otra parte, en el artículo 139 se expresa que la calificación final de los estudiantes en cada una de las asignaturas, se determinará mediante la combinación de la calificación obtenida en la respectiva prueba con la "nota de presentación", que se define como el promedio de las calificaciones obtenidas en la Educación Diversificada en: Estudios Sociales y Cívica, Español, Inglés o Francés, Matemáticas y Biología, Química o Física (según corresponda). La combinación señalada se realiza de la siguiente manera:

a. La nota de presentación correspondiente al promedio de las calificaciones constituirá el $40 \%$ del total de la calificación final de la asignatura.

b. La calificación obtenida en la respectiva prueba de bachillerato tendrá un valor de $60 \%$ del total de la calificación final de la asignatura.

Los estudiantes que obtengan una calificación final igual o superior a setenta se tendrán por aprobados en la respectiva asignatura. 
La calificación de los aprendizajes del estudiante en cada asignatura, según el artículo 30, inciso h, es el resultado de la suma de los siguientes componentes:

$\begin{array}{ll}\square & \text { Trabajo cotidiano: } 10 \% \\ \square & \text { Trabajo extraclase: } 10 \% \\ \square & \text { Pruebas (mínimo dos): } 70 \% \\ \square & \text { Concepto: } 5 \% \\ \square & \text { Asistencia: } 5 \%\end{array}$

Finalmente, en el Informe nacional de bachillerato 1997 (MEP: 5) se señaló que en el ámbito internacional se ha generado la necesidad de instaurar controles de calidad del sistema educativo para cuantificar su eficacia y brindar información para la toma de decisiones. En nuestro país esta tendencia se ha fortalecido y se define como una de las estrategias para efectuar un control de salida, con el fin de mejorar y definir la calidad de la educación costarricense; puesto que las pruebas de bachillerato, como instrumentos de medición, permiten:

- acreditar el dominio de los conocimientos básicos

- brindar información para la toma de decisiones y realimentar el proceso educativo.

\section{Justificación}

De acuerdo con el informe de Bachillerato 1998 y los datos de los cuadros 1 y 2 , se han presentado diferencias entre la nota de bachillerato y la del examen. Según el informe supracitado, se puede deber a que la nota de presentación es el promedio de todas las asignaturas del plan de estudio de la Educación Diversificada y, generalmente, las calificaciones de las materias no medidas en las pruebas de bachillerato son altas, lo que produce un incremento en la nota de presentación, que en conjunto con el examen, conforman la puntuación final de bachillerato.
A raíz de esta diferencia, surgió la inquietud de comprobar si existe o no alguna relación entre las calificaciones de las asignaturas de Bachillerato en Educación Media: Matemática, Español, Inglés, Francés, Estudios Sociales, Biología, Física y Química con sus respectivos exámenes de acreditación.

Los promedios de Bachillerato en Educación Media de los estudiantes han variado en los últimos años, según la modalidad de los colegios, como se muestra en el siguiente cuadro:

\section{Cuadro 1}

Promedios de las notas de bachillerato por año, según modalidad de los colegios

\begin{tabular}{lcccc}
\hline \multicolumn{5}{c}{ AÑOS } \\
\hline MODALIDAD & 1995 & 1996 & 1997 & 1998 \\
\hline Académicos & 78,57 & 74,75 & 79,53 & 80,30 \\
Nocturnos & 69,84 & 59,59 & 70,89 & 71,61 \\
Técnicos & 73,02 & 66,42 & $76,02(1)$ & $77,62(1)$ \\
& & & $74,49(2)$ & $69,32(2)$ \\
\hline
\end{tabular}

(1) Opción 1: Son estudiantes de duodécimo nivel.

(2) Opción 2: Son estudiantes de undécimo año.

También la media aritmética de las calificaciones en los exámenes ha variado por año y modalidad. Los colegios nocturnos obtuvieron los promedios más bajos, como se muestra en el cuadro 2.

Cuadro 2

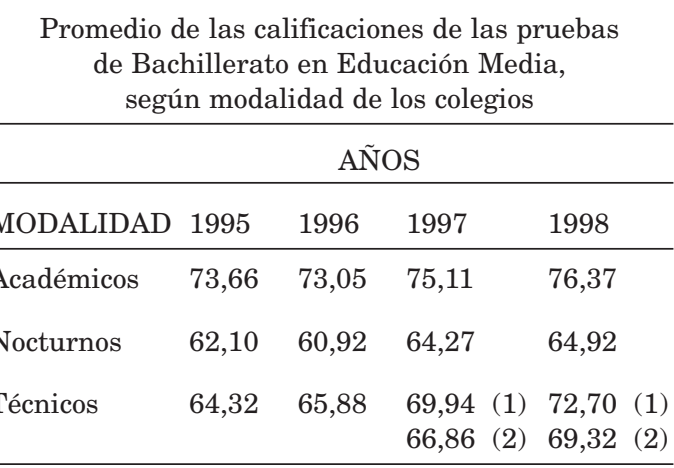

(1) Opción 1: Son estudiantes de duodécimo nivel.

(2) Opción 2: Son estudiantes de undécimo año. 


\section{Estado de la cuestión}

Una función básica de las pruebas de aprovechamiento, de acuerdo con Aiken, es determinar cuánto sabe una persona acerca de cierto tema o qué tan bien pueden poner en práctica ciertas habilidades. Los resultados de este tipo de pruebas informan a los alumnos, docentes, administrativos y padres de familia, acerca de los logros y deficiencias académicas de los estudiantes. También subraya este autor, que las calificaciones no constituyen el único medio para evaluar la efectividad de la enseñanza, pero sí ofrecen una medida de la calidad de la educación y, de esa forma, contribuyen a su mejoramiento ${ }^{3}$.

$\mathrm{Al}$ respecto Popham considera que las pruebas estandarizadas de logro muestrean los conocimientos o destrezas de un dominio de contenidos. Generalmente, este instrumento de medición proporciona interpretaciones válidas, referida a normas, sobre la situación de un estudiante respecto a una porción sustancial de contenidos. Además, si se asume que el grupo normativo (promedio) nacional es genuinamente representativo del país, los educadores y padres pueden hacer inferencias útiles sobre los estudiantes ${ }^{4}$.

De acuerdo con este autor, una de las principales inferencias se refiere a las fortalezas y debilidades relativas de los estudiantes entre las distintas asignaturas y entre las diversas áreas o temas de cada una de las asignaturas; aunque estas pruebas contienen pocos ítemes como para permitir una comparación significativa en el interior de una misma asignatura. Un segundo tipo de inferencia se relaciona con el mejoramiento del estudiante a través del tiempo en diferentes asignaturas.

Asimismo, Popham considera que emplear las pruebas estandarizadas de logros para evaluar la calidad de la educación es como medir la temperatura con una cuchara; ya que estos instrumentos tienen la misión de medir algo distinto y deberían usarse para hacer las interpretaciones comparativas que se supone deben suministrar.

Desde una perspectiva amplia, la calidad de la educación, según Hilda Lanza, incluye tanto los aspectos referidos al logro de niveles equitativos de cobertura, como a la distribución de conocimientos socialmente significativos para el conjunto de la población ${ }^{5}$. Además, señala los tres grandes ejes que están incidiendo en la disminución de la calidad de nuestros sistemas educativos regionales:

- La baja significatividad de los conocimientos.

- La inequidad en la prestación de los servicios educativos.

- La baja racionalidad y eficiencia en la gestión institucional.

En este sentido, la autora indica que en los países iberoamericanos existe un desafío: producir una información que respete la relación entre la vida cotidiana de las escuelas y los registros de tipo estadístico que proporcionan la imagen de la realidad educativa en el nivel del sistema educativo.

En este estudio se recolectó información acerca del logro académico de los estudiantes en las pruebas de Bachillerato en Educación Media y, en general, del comportamiento del sistema educativo en su conjunto. Ahora bien, esta información constituye una cara de la moneda, puesto que igualmente importante es conocer los factores procesuales y cualitativos que subyacen en los resultados de estas pruebas.

Asimismo, Lanza destaca que el papel de realimentación de la evaluación se logra a través de la difusión responsable de los resultados a:

- Los centros educativos para fortalecer la responsabilidad institucional sobre los resultados de la gestión escolar.

- Los docentes para que generen nuevas estrategias didácticas $\mathrm{y}$ 
metodológicas para mejorar el aprendizaje de los estudiantes.

- Las familias para que confronten sus opiniones acerca del desempeño de las instituciones y para tomar decisiones más responsables en la elección de alternativas educativas.

- Las autoridades y actores políticos para la toma de decisiones y búsqueda de soluciones efectivas para la capacitación docente, las acciones de las políticas compensatorias y la producción de recomendaciones para mejorar la tarea en las aulas.

\section{Limitaciones}

Se debe destacar que existen múltiples factores que incidieron en los resultados de las pruebas; sin embargo, en este estudio se asumió que las condiciones exógenas y endógenas de los estudiantes no han cambiado significativamente entre el período de formación y preparación en la Educación Diversificada y en el momento de aplicación de las pruebas; por consiguiente, no se analizaron las condiciones inherentes al logro académico de los estudiantes en el Bachillerato en Educación Media.

Otra limitación consistió en que el promedio total de las asignaturas fue producto de diversos componentes: pruebas, trabajo cotidiano, extraclase, asistencia y concepto y éste se asoció con los resultados de cada prueba; por lo tanto, los dos conjuntos de medidas no fueron equiparables.

Finalmente las pruebas de bachillerato son estandarizadas y difieren en diversos aspectos de los exámenes elaborados por los docentes.

\section{Procedimientos metodológicos}

\section{Tipo de estudio}

El diseño de esta evaluación fue no experimental, de tipo transversal con un enfoque correlacional; según Ary y otros, es un tipo de investigación descriptiva que se utiliza para medir el grado de asociación existente entre las variables y permite indagar hasta qué punto las alteraciones de una dependen de los cambios de otra. La magnitud de la relación se calcula mediante un coeficiente de correlación que se expresa como un índice numérico ${ }^{6}$.

Para Hopkins y otros, los coeficientes de correlación resumen la magnitud y la dirección de una asociación entre dos variables. La primera se indica con un valor absoluto, así cuanto más alto sea, mayor es la relación y la predicción de $\mathrm{Y}$ a partir de $\mathrm{X}$ o viceversa; mientras que la segunda se marca por los signos (+ 0 -). Una asociación positiva indica una tendencia a mantener valores similares en las dos medidas; es decir, las puntuaciones altas están asociadas con las altas y las bajas con las bajas. Una relación negativa indica que quienes calificaron alto en una variable $\mathrm{X}$ tienden a calificar bajo en una variable $\mathrm{Y}$ o viceversa. Además, apuntan estos autores, que los valores diferentes de cero indican que Y puede predecirse, con mayor o menor precisión, si conocemos X; pero la predicción por sí misma no presupone una relación causal 7 .

$\mathrm{Al}$ respecto, Runyon y Haber señalan que el término predicción en Estadística no implica ninguna deducción hacia el futuro, sino que se refiere al uso de la información de una variable para obtener información respecto de otra ${ }^{8}$.

Cuando se encuentran correlaciones bajas, según estos autores, se cae en la tentación de concluir que la asociación es débil o no existe ninguna relación. Esta falta de evidencia puede deberse a que: las dos variables no están relacionadas o la relación no es lineal ${ }^{9}$.

En este estudio se utilizó el coeficiente de correlación producto momento ( $\mathrm{r}$ de Pearson) porque las variables medidas corresponden a una escala de intervalos (el punto cero es arbitrario). 


\section{Problema}

¿Existe relación entre los resultados en las pruebas de Bachillerato en Educación Media con los promedios de las asignaturas fundamentales de la Educación Diversificada?

\section{Objetivo metodológico}

Medir el grado de asociación entre el promedio de calificaciones de la Educación Diversificada en Matemática, Español, Estudios Sociales, Inglés, Francés, Biología, Química y Física con los respectivos resultados de las pruebas de Bachillerato en Educación Media.

\section{Definiciones sustantivas de las variables}

\section{Logro académico:}

El dominio de conocimientos esenciales definidos por el currículum nacional de la Educación Diversificada y concretados en los temarios de Bachillerato en Educación Media.

Promedios de las asignaturas

fundamentales:

Las calificaciones obtenidas por los estudiantes en Español, Inglés o Francés, Matemáticas, Estudios Sociales, Biología o Física o Química en décimo, undécimo y duodécimo (del último nivel sólo se consignan las calificaciones correspondientes al primer y segundo período), de acuerdo con los siguientes componentes: trabajo cotidiano (10\%), extraclase (10\%), pruebas (70\%), concepto $(5 \%)$ y asistencia $(5 \%)$.

\section{Definiciones operacionales}

Logro académico:

La nota obtenida por los estudiantes en las pruebas de Bachillerato en Educación Media.

\section{Promedios de las asignaturas fundamentales}

La media aritmética de las calificaciones obtenidas por los estudiantes en las asignaturas fundamentales durante la Educación Diversificada.

\section{Población}

Esta evaluación se realizó con la población de estudiantes de Bachillerato en Educación Media de la convocatoria ordinaria del año 1999, como se muestra en el siguiente cuadro.

Asignatura Población

\begin{tabular}{lr}
\hline Biología & 12384 \\
Química & 2644 \\
Física & 1972 \\
Inglés & 15518 \\
Francés & 1471 \\
Español & 17124 \\
Estudios sociales & 17172 \\
Matemáticas & 17185 \\
\hline
\end{tabular}

\section{Análisis estadísticos}

El análisis de las pruebas de Bachillerato en Educación Media de la convocatoria ordinaria de 1999 se realizó con la población; aunque se excluyeron los casos con datos incompletos (vg. nota de presentación).

Una parte del análisis presentó los porcentajes de estudiantes aprobados en cada prueba según el sexo, la zona, el tipo y la modalidad del colegio. Para la condición de aprobado se sumó el resultado de la prueba (con un valor de $60 \%$ en los colegios académicos diurnos y técnicos y de $56 \%$ en los nocturnos) con la nota de presentación global de la Educación Diversificada (con un valor de $40 \%$ en los colegios académicos diurnos y en los técnicos, y de $44 \%$ en los nocturnos). 
La otra parte fue la correlación del resultado de la prueba (sin la nota de presentación) con el promedio de las calificaciones de la Educación Diversificada correspondientes a cada asignatura; por ejemplo, se asoció la nota de la prueba de Biología con el promedio de las calificaciones de la Educación Diversificada de esa asignatura. Se utilizó el coeficiente de correlación de Pearson, pues es una medida del grado en que los mismos sujetos ocupan una posición relativa respecto a dos variables, que en este análisis, corresponden a una escala de intervalos: el cero es arbitrario y no representa la ausencia de conocimientos, representa un punto conveniente del cual se marcan intervalos de igual magnitud.

Para Hopkins et al..$^{10}$ las variables perfectamente correlacionadas $(r=+1.0$ ó $r=-1.0$ ), una calificación en una de las variables dará una predicción perfectamente precisa del valor de la otra variable. Para variables fuertemente asociadas, dará una predicción bastante precisa del valor de la otra y finalmente para las correlaciones débiles, un valor para una de las variables da una predicción del valor de la otra, que es un poco mejor que una adivinación directa.

Además, señalan estos autores que el coeficiente de correlación algunas veces se confunde con un porcentaje. Por ejemplo una $\mathrm{r}$ de .60 significa que hay una tendencia para los pares de valores de cada unidad de ser algo similar, pero con muchas excepciones; no significa que hay un $60 \%$ de acuerdo, conformidad, correspondencia, congruencia o consistencia entre los valores, ni que las dos variables están $60 \%$ relacionadas, ni que el $60 \%$ de lo que mide $\mathrm{X}$ también es medido por $\mathrm{Y}$.

Finalmente destacan que si las excepciones a la regla son pocas y pequeñas, la magnitud de $\mathrm{r}$ es alta, quizá .90 o superior. Cuando con frecuencia hay excepciones sustanciales a la regla, la magnitud será moderada, quizá en la proximidad de 0.50 o por abajo. Cuando hay muchas excepciones evidentes a la regla, la magnitud de $r$ puede ser completamente pequeña, quizá sólo .30 o por abajo.

\section{Biología}

El promedio de la nota de la prueba de Biología fue un 75, mientras que el de las calificaciones de la Educación Diversificada fue un 82 y la desviación estándar (medida que refleja la dispersión de las calificaciones) de las notas de la prueba de Biología fue mayor $(12,2)$, con respecto a las del colegio $(7,7)$.

De un total de 7124 estudiantes masculinos y 5260 estudiantes femeninos que realizaron la prueba de Biología, el $86 \%$ y $84 \%$ respectivamente, aprobaron la prueba y, a pesar de la desigualdad numérica (1 297 de colegios privados, 10397 de públicos y 690 de subvencionados privados), no hubo diferencias entre la promoción de privados (95\%) y subvencionados privados (95\%); mientras que los colegios públicos obtuvieron el porcentaje más bajo (83\%), aunque representó el $82 \%$ de la promoción total en Biología (12\% los privados y $6 \%$ los subvencionados privados).

Según la modalidad de los colegios, los nocturnos obtuvieron la más baja promoción (68\%) y la más alta, un $89 \%$ en los académicos diurnos.

La categorización urbano y rural fue establecida en el año 1984 (año del censo anterior al 2 000) por el Instituto Nacional de Estadística y Censos; por lo tanto, los colegios se clasificaron de acuerdo con su ubicación territorial. En la convocatoria ordinaria de 1999 cerca del $20 \%$ de los estudiantes procedían de colegios ubicados en una zona rural, con un $81 \%$ de aprobación y aproximadamente el $80 \%$ en zonas urbanas, con un $86 \%$ de promoción. 
Coeficiente de correlación Pearson

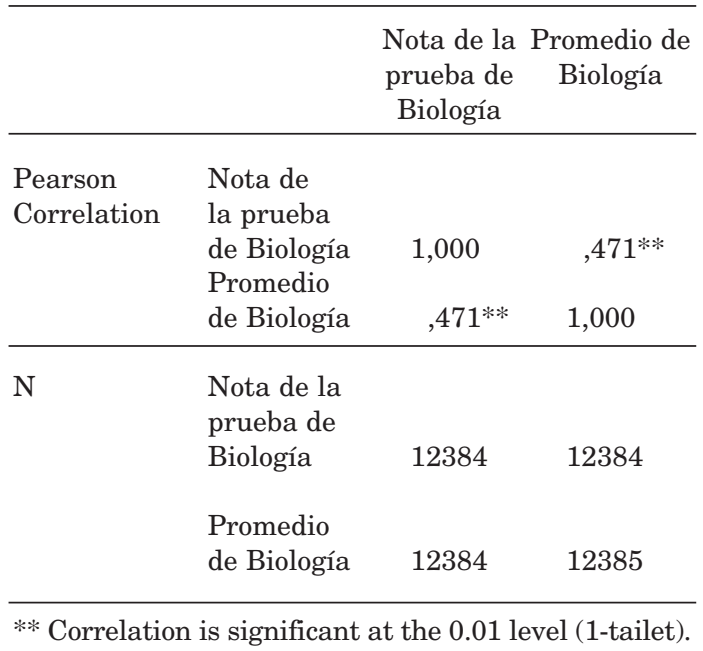

Resultó una correlación moderada: 0.47 y con dirección positiva, lo que significó que los estudiantes con altas calificaciones en el colegio en Biología tendieron a obtener notas altas en el examen, con frecuentes excepciones a la regla. Además, la correlación fue significativa en el nivel 0,01 con un examen estadístico unilateral.

\section{Química}

El promedio de los resultados de la prueba de Química fue de 76 y 84 el de las calificaciones del colegio. Al igual que en Biología, las calificaciones de las pruebas mostraron una mayor dispersión con respecto al promedio.

Los porcentajes de aprobación fueron semejantes en los estudiantes femeninos $(86 \%)$ y masculinos (88\%). Al igual que en Biología, en los colegios públicos se presentaron los porcentajes más bajos de aprobación (83\%), aunque la mayor cantidad de estudiantes pertenecía a esta modalidad (1 813). En los privados y los subvencionados privados aprobaron el $95 \% \mathrm{y}$ $98 \%$, respectivamente.

La tendencia de menor aprobación se mantuvo en los colegios nocturnos
(56\%); mientras que los académicos diurnos $(89 \%)$ y técnicos (91\%) obtuvieron resultados muy semejantes.

Coeficiente de correlación

\begin{tabular}{llcc}
\hline & \multicolumn{3}{c}{$\begin{array}{c}\text { Nota de la Promedio de } \\
\text { prueba de } \\
\text { Química }\end{array}$} \\
\hline $\begin{array}{l}\text { Pearson } \\
\text { Correlation }\end{array}$ & $\begin{array}{l}\text { Nota de } \\
\text { la prueba } \\
\text { de Química }\end{array}$ & 1,000 &, $473^{* *}$ \\
& $\begin{array}{l}\text { Promedio } \\
\text { de Química }\end{array}$ &, $473^{* *}$ & 1,000 \\
\hline $\mathrm{N}$ & $\begin{array}{l}\text { Nota de la } \\
\text { prueba de } \\
\text { Química }\end{array}$ & 2643 & 2643 \\
& $\begin{array}{l}\text { Promedio } \\
\text { de Química }\end{array}$ & 2643 & 2644 \\
\hline
\end{tabular}

** Correlation is significant at the 0.01 level (1-tailet).

El grado de asociación entre los resultados de la prueba de Química con las respectivas calificaciones de la Educación Diversificada fue moderado: 0.47. La dirección fue positiva, por lo tanto los estudiantes con altas calificaciones en Química tendieron a obtener resultados altos en la prueba, pero con frecuentes excepciones a la regla.

Además, la correlación fue significativa en el nivel 0,01 con un examen estadístico unilateral.

\section{Física}

Se presentó una diferencia entre el promedio de la prueba de Física (75) y el de las calificaciones de la Educación Diversificada (84). Además, las notas de las pruebas mostraron una mayor variabilidad $(12,8)$ con respecto al promedio.

En esta asignatura los estudiantes masculinos (88\%) lograron una mejor promoción que las mujeres $(81 \%)$ y, al igual que en las otras ciencias, el rendimiento más bajo se presentó en los colegios públicos 
(80\%); aunque la mayor cantidad de estudiantes pertenecía a esta modalidad. En los colegios privados fue de un $95 \%$ y en los subvencionados privados, un $93 \%$.

Los colegios de zona rural lograron un mejor rendimiento; mientras que en la modalidad nocturna se manifestó la tendencia de menor aprobación (77\%). En los técnicos se logró el 100\% de aprobación de un total de cuatro estudiantes y en los académicos diurnos un $87 \%$.

Coeficiente de correlación

\begin{tabular}{llcc}
\hline & & $\begin{array}{c}\text { Nota de la } \\
\text { prueba de } \\
\text { Física }\end{array}$ & $\begin{array}{c}\text { Promedio de } \\
\text { Física }\end{array}$ \\
\hline $\begin{array}{l}\text { Pearson } \\
\text { Correlation }\end{array}$ & $\begin{array}{l}\text { Nota de } \\
\text { la prueba } \\
\text { de Física }\end{array}$ & 1,000 &, $515^{* *}$ \\
& $\begin{array}{l}\text { Promedio } \\
\text { de Física }\end{array}$ &, $515^{* *}$ & 1,000 \\
\hline $\mathrm{N}$ & $\begin{array}{l}\text { Nota de la } \\
\text { prueba de }\end{array}$ & 1972 & 1972 \\
& $\begin{array}{l}\text { Física } \\
\text { Promedio } \\
\text { de Física }\end{array}$ & 1972 & 1972 \\
\hline
\end{tabular}

** Correlation is significant at the 0.01 level (1-tailet).

La prueba de Física se asoció moderadamente (0.51). La dirección positiva indicó que los estudiantes con altos promedios en el colegio tendieron a obtener altas calificaciones en la prueba de Física, aunque con frecuentes excepciones a la regla.

Asimismo, la correlación fue significativa en el nivel 0,01 con un examen estadístico unilateral.

\section{Inglés}

Tanto las calificaciones de la Educación Diversificada como la prueba de Inglés obtuvieron un promedio muy semejante (81 y 82 respectivamente), aunque las notas del examen presentaron mayor variabilidad con respecto al promedio $(11,7)$.
Los porcentajes de aprobación fueron casi iguales entre los hombres y las mujeres, $96 \%$ y $95 \%$ respectivamente. Tampoco hubo gran diferencia entre las tres modalidades de colegio, pese a que en los públicos estaba la mayor cantidad de estudiantes, el $94 \%$ aprobaron Inglés y el 99\% en las otras dos modalidades.

Asimismo, los porcentajes de aprobación fueron muy parecidos en los colegios académicos diurnos (96\%), nocturnos (91\%) y técnicos $(94 \%)$.

Coeficiente de correlación

\begin{tabular}{llcc}
\hline & & $\begin{array}{c}\text { Nota de la } \\
\text { prueba de } \\
\text { Ingles }\end{array}$ & $\begin{array}{c}\text { Promedio de } \\
\text { Ingles }\end{array}$ \\
\hline $\begin{array}{l}\text { Pearson } \\
\text { Correlation }\end{array}$ & $\begin{array}{l}\text { Nota de } \\
\text { la prueba } \\
\text { de Inglés }\end{array}$ & 1,000 &, $475^{* *}$ \\
& $\begin{array}{l}\text { Promedio } \\
\text { de Inglés }\end{array}$ &, $475^{* *}$ & 1,000 \\
\hline $\mathrm{N}$ & $\begin{array}{l}\text { Nota de la } \\
\text { prueba de }\end{array}$ & & \\
Inglés & 15517 & 15517 \\
& $\begin{array}{l}\text { Promedio } \\
\text { de Inglës }\end{array}$ & 15517 & 15518 \\
\hline
\end{tabular}

** Correlation is significant at the 0.01 level (1-tailet).

La dirección de la correlación fue positiva y moderada: 0.47 ; lo que significó que estudiantes con altas calificaciones en el colegio tendieron a obtener notas altas en la prueba de Inglés, aunque con frecuentes excepciones.

Además, la correlación fue significativa en el nivel 0,01 con un examen estadístico unilateral.

\section{Francés}

El promedio de las calificaciones de la Educación Diversificada y de la nota de la prueba de Francés fue igual: un 82. La diferencia se presentó en una mayor desviación estándar de los resultados de la 
prueba $(9,4)$ con respecto a las calicaciones del colegio $(7,6)$.

En relación con la promoción prevalecieron los porcentajes altos en esta asignatura, por ejemplo, los estudiantes femeninos y masculinos lograron un 97\%; al igual que en los colegios públicos; mientras que en los privados y los subvencionados privados fue de un $100 \%$.

Asimismo, de 1299 estudiantes que presentaron en los diurnos, un 98\% aprobó y en los nocturnos, fue un $89 \%$ de un total de 171 estudiantes.

Coeficiente de correlación

\begin{tabular}{llcc}
\hline & & $\begin{array}{c}\text { Nota de la } \\
\text { prueba de } \\
\text { Francés }\end{array}$ & $\begin{array}{c}\text { Promedio de } \\
\text { Francés }\end{array}$ \\
\hline $\begin{array}{l}\text { Pearson } \\
\text { Correlation }\end{array}$ & $\begin{array}{l}\text { Nota de } \\
\text { la prueba } \\
\text { de Francés }\end{array}$ & 1,000 &, $402^{* *}$ \\
& $\begin{array}{l}\text { Promedio } \\
\text { de Francés }\end{array}$ &, $402^{* *}$ & 1,000 \\
\hline $\mathrm{N}$ & $\begin{array}{l}\text { Nota de la } \\
\text { prueba de } \\
\text { Francés }\end{array}$ & 1471 & 1471 \\
& $\begin{array}{l}\text { Promedio } \\
\text { de Francés }\end{array}$ & 1471 & 1471 \\
& & \\
\hline
\end{tabular}

** Correlation is significant at the 0.01 level (1-tailet).

La correlación fue moderada: $0.40 \mathrm{y}$ con dirección positiva; lo que indicó que los estudiantes con promedios altos en el colegio tendieron a obtener altas calificaciones en la prueba de Francés, aunque con frecuentes excepciones.

También la correlación fue significativa en el nivel 0,01 con un examen estadístico unilateral.

\section{Español}

En esta asignatura fue el mismo promedio en la prueba y en las notas de la Educación Diversificada (79); así como la misma dispersión de las calificaciones $(7,9)$ con respecto al promedio.

$\mathrm{Al}$ igual que en otras pruebas, no hubo gran diferencia entre hombres (96\%) y mujeres (98\%) en la promoción; ni por modalidad, pues en los subvencionados privados y privados fue un $100 \%$ y $96 \%$ en públicos; aunque en estos últimos se ubicó la mayoría de los estudiantes (13 489, equivalente a un $79 \%$ ).

En los colegios nocturnos se mantuvo la tendencia de baja aprobación (83\%); mientras que en los académicos diurnos aprobaron un $99 \%$ y en los técnicos, un $98 \%$.

Coeficiente de correlación

\begin{tabular}{llcc}
\hline & & $\begin{array}{c}\text { Nota de la } \\
\text { prueba de } \\
\text { Español }\end{array}$ & $\begin{array}{c}\text { Promedio de } \\
\text { Español }\end{array}$ \\
\hline $\begin{array}{l}\text { Pearson } \\
\text { Correlation }\end{array}$ & $\begin{array}{l}\text { Nota de } \\
\text { la prueba } \\
\text { de Español }\end{array}$ & 1,000 &, $379^{* *}$ \\
& $\begin{array}{l}\text { Promedio } \\
\text { de Español }\end{array}$ &, $379^{* *}$ & 1,000 \\
\hline $\mathrm{N}$ & $\begin{array}{l}\text { Nota de la } \\
\text { prueba de } \\
\text { Español }\end{array}$ & 17123 & 17123 \\
& $\begin{array}{l}\text { Promedio } \\
\text { de Español }\end{array}$ & 17123 & 17124 \\
\hline
\end{tabular}

** Correlation is significant at the 0.01 level (1-tailet).

El grado de asociación fue moderado: 0.38 con dirección positiva; por lo tanto, los estudiantes con calificaciones altas en el colegio tendieron a obtener notas altas en la prueba de Español, pero con excepciones evidentes.

Además, la correlación fue significativa en el nivel 0,01 con un examen estadístico unilateral.

\section{Estudios Sociales}

El promedio de la prueba fue 73 y 80 el de las calificaciones de Educación 
Diversificada. Al igual que en las otras asignaturas, las notas de las pruebas se dispersaron más del promedio.

El $46 \%$ de los estudiantes eran masculinos con un $88 \%$ de aprobación y las mujeres con un $81 \%$, y en los públicos se presentó el valor más bajo (82\%); sin embargo, se ha de considerar que la mayoría de los estudiantes (79\%) provenían de estos colegios; en los privados (95\%) y los subvencionados privados (96\%) fue la promoción casi igual.

En esta asignatura los colegios nocturnos obtuvieron el más bajo porcentaje de aprobación (58\%); mientras que los técnicos $(84 \%)$ y diurnos (89\%) lograron un nivel de aprobación muy semejante.

\begin{tabular}{|c|c|c|c|}
\hline \multicolumn{4}{|c|}{ Coeficiente de correlación } \\
\hline & & $\begin{array}{c}\text { Nota de la } \\
\text { prueba de } \\
\text { Estudios } \\
\text { Sociales }\end{array}$ & $\begin{array}{c}\text { Promedio de } \\
\text { Estudios } \\
\text { Sociales }\end{array}$ \\
\hline \multirow[t]{2}{*}{$\begin{array}{l}\text { Pearson } \\
\text { Correlation }\end{array}$} & $\begin{array}{l}\text { Nota de } \\
\text { la prueba } \\
\text { de Estudios } \\
\text { Sociales }\end{array}$ & 1,000 &, $442^{* *}$ \\
\hline & $\begin{array}{l}\text { Promedio } \\
\text { de Estudios } \\
\text { Sociales }\end{array}$ &, $442^{* *}$ & 1,000 \\
\hline \multirow[t]{2}{*}{$\mathrm{N}$} & $\begin{array}{l}\text { Nota de la } \\
\text { prueba de } \\
\text { Estudios } \\
\text { Sociales }\end{array}$ & 17171 & 17171 \\
\hline & $\begin{array}{l}\text { Promedio } \\
\text { de Estudios } \\
\text { Sociales }\end{array}$ & 17171 & 17172 \\
\hline
\end{tabular}

** Correlation is significant at the 0.01 level (1-tailet).

El grado de asociación entre la prueba con las calificaciones de la Educación Diversificada en Estudios Sociales fue moderado: 0.44 ; por consiguiente los estudiantes con altas notas en el colegio tendieron a obtener buenas calificaciones en la prueba, pero con frecuentes excepciones.
La correlación resultó significativa en el nivel 0,01 con un examen estadístico unilateral.

\section{Matemáticas}

En esta asignatura se presentó la mayor variabilidad $(17,5)$ en las notas de la prueba con respecto al promedio: 68 , el más bajo de todas las pruebas. Asimismo, hubo una gran diferencia con respecto al promedio de las calificaciones del colegio: 79, con una desviación estándar de 8,4.

Aunque eran más mujeres, solo el 65\% aprobó Matemáticas, mientras que los estudiantes masculinos lograron un porcentaje más alto de aprobación (70\%). También se mostró una gran diferencia en la promoción en los colegios públicos $(61 \%)$ con respecto a los privados $(90 \%)$ y los subvencionados privados $(92 \%)$.

Definitivamente, la prueba de Matemáticas marcó diferencias importantes, aunque fue la asignatura con más bajo porcentaje de aprobación en las tres modalidades. En los nocturnos se presentó la situación más crítica, sólo aprobaron el $35 \%$ de los estudiantes; en los técnicos un $57 \%$ y en los académicos diurnos un $75 \%$.

Coeficiente de correlación

\begin{tabular}{|c|c|c|c|}
\hline & $\begin{array}{r}\mathrm{N} \\
\mathrm{pr} \\
\mathrm{Ma}\end{array}$ & $\begin{array}{l}\text { ta de la } \\
\text { ueba de } \\
\text { emáticas }\end{array}$ & $\begin{array}{l}\text { Promedio de } \\
\text { Matemáticas }\end{array}$ \\
\hline \multirow[t]{2}{*}{$\begin{array}{l}\text { Pearson } \\
\text { Correlation }\end{array}$} & $\begin{array}{l}\text { Nota de } \\
\text { la prueba } \\
\text { de Matemáticas }\end{array}$ & 1,000 &, $520 * *$ \\
\hline & $\begin{array}{l}\text { Promedio } \\
\text { de Matemáticas }\end{array}$ &, $520 * *$ & 1,000 \\
\hline \multirow[t]{2}{*}{$\mathrm{N}$} & $\begin{array}{l}\text { Nota de la } \\
\text { prueba de } \\
\text { Matemáticas }\end{array}$ & 17184 & 17184 \\
\hline & $\begin{array}{l}\text { Promedio } \\
\text { de Matemáticas }\end{array}$ & 17184 & 17185 \\
\hline
\end{tabular}

** Correlation is significant at the 0.01 level (1-tailet). 
Pese a la gran variabilidad y diferencias en los resultados de la prueba de Matemáticas, fue la correlación más alta con respecto a las otras asignaturas: 0.52 ; lo que significó que los estudiantes buenos en Matemáticas tendieron a obtener altas calificaciones en la prueba, aunque con frecuentes excepciones.

Además, la correlación fue significativa en el nivel 0,01 con un examen estadístico unilateral.

Finalmente, se realizó una comparación de los porcentajes de aprobados por asignatura; considerando, por un lado, la nota de presentación total de la Educación Diversificada (vigente) calculada con todas las asignaturas $\mathrm{y}$, por otro, una base que incluye únicamente los promedios de las cinco asignaturas medidas en las pruebas. La condición de aprobado se obtuvo al sumar el resultado de la prueba (con un valor de $60 \%$ en los colegios académicos diurnos y técnicos y de $56 \%$ en los nocturnos) con la nota de presentación (con un valor de $40 \%$ en los colegios académicos diurnos y en los técnicos y de $44 \%$ en los nocturnos).

\begin{tabular}{lcc}
\hline Asignaturas & \multicolumn{2}{c}{$\begin{array}{c}\text { Porcentajes de } \\
\text { Aprobación }\end{array}$} \\
\hline Total & Base \\
Biología & 84,7 & 77,7 \\
Español & 96,9 & 92,6 \\
Estudios Sociales & 84,5 & 76,8 \\
Física & 85,7 & 79,4 \\
Francés & 97,3 & 94,6 \\
Inglés & 95,1 & 90,5 \\
Matemáticas & 67,2 & 60,8 \\
Química & 87,1 & 81,5 \\
Porcentaje de & & \\
Promoción & & \\
\hline
\end{tabular}

Total: Nota de presentación global.

Base: Nota de presentación base.

De acuerdo con estos cálculos, se evidenció que los porcentajes de aprobación, en todas las asignaturas, bajaron significativamente al excluir de la nota de presentación los promedios de las asignaturas no medidas en el Bachillerato de Educación Media.

Además, se estimó el porcentaje de promoción considerando únicamente las cinco asignaturas medidas en las pruebas. Según los datos, 1401 estudiantes $(8,1 \%)$ no hubieran aprobado el bachillerato utilizando la nota de presentación base.

\section{Conclusiones}

El principal objetivo de este estudio era determinar el grado de asociación de cada una de las pruebas de Bachillerato en Educación Media con los respectivos promedios de las calificaciones de la Educación Diversificada, sobresaliendo los siguientes resultados:

- $\quad$ En todas las asignaturas el coeficiente de correlación fue moderado, alrededor de 0.50; el más bajo fue en Español (0.38); en otros términos, se comprobó que ambas puntuaciones (pruebas y promedios) estaban relacionadas o tendieron a variar conjuntamente.

- Las correlaciones fueron positivas, lo que significó que los estudiantes con altas calificaciones en la Educación Diversificada tendieron a obtener notas altas en las pruebas, aunque con frecuentes excepciones por el grado de asociación.

- $\quad$ En los colegios nocturnos se presentaron los porcentajes más bajos de aprobación en todas las asignaturas, particularmente en Matemáticas; debido posiblemente a sus desventajas sociales y educativas con respecto a los estudiantes de las otras modalidades.

- Los porcentajes de aprobación por sexo fueron muy semejantes, aunque en Matemáticas, Física y Estu- 
dios Sociales las mujeres obtuvieron porcentajes inferiores, con cinco o siete puntos porcentuales menos.

- Los colegios privados y subvencionados privados alcanzaron un nivel de aprobación muy parecido; mientras que los resultados de los públicos eran los más bajos; sin embargo, se ha de considerar que cerca del $80 \%$ de los estudiantes provenían de estos colegios. Además, se debe contemplar que estas instituciones han estado en una evidente desventaja en múltiples condiciones como: infraestructura, equipamiento, gestión administrativa, recursos humanos y económicos.

- No se presentaron grandes diferencias en los resultados entre los colegios de la zona urbana y la rural; pese a sus diferencias culturales y geográficas.

- Las calificaciones de las pruebas de bachillerato mostraron mayor dispersidad con respecto al promedio. Mientras que las calificaciones de cada asignatura durante la Educación Diversificada se desviaron menos del promedio; es decir, fueron más constantes. Esta diferencia podría deberse a que los estudiantes durante su período formativo desarrollaron un sentido personal y social que marcó sus relaciones con el centro educativo y con el cumplimiento de sus responsabilidades académicas; por consiguiente, esto explicaría, en cierta medida, la constancia de sus calificaciones.

- Finalmente, es importante destacar que si la nota de presentación se hubiera calculado con sólo las cinco asignaturas medidas en las pruebas, 1 401 estudiantes $(8,1 \%)$ no hubieran logrado la condición de promovidos en el Bachillerato en Educación Media.

\section{Notas}

1. MEP. Informe nacional de resultados de Bachillerato 1998. San José: MEP, 1999, pp. 2-4.

2. MEP. Reglamento de Evaluación de los Aprendizajes. San José: MEP. 2000.

3. Lewis Aiken. Test psicológicos y evaluación. México: Prentice Hall Hispanoamerica, S.A. 1996, pág. 109.

4. Popham, W. James. “¿Por qué las pruebas estandarizadas no miden la calidad educativa?"

5. Hilda Lanza. La evaluación de los sistemas educativos en Iberoamérica: algunas reflexiones en torno a su especificidad. Madrid: Ponencia en el Congreso Internacional de Evaluación de la Calidad. 1996.

6. Donald Ary et al. Introducción a la investigación pedagógica. México: McGraw-Hill. 1998, pág. 318.

7. Kenneth D. Hopkins et al. Estadística Básica para las Ciencias Sociales y del Comportamiento. México: Prentice-Hall Hispanoamericana, S.A. 1997, pág. 100.

8. Runyon, R. y Haber, A. Estadística para las ciencias sociales. Estados Unidos: AddisonWesley Iberoamericana, S.A. 1992, pág. 148.

9. $\quad$ Idem, pág. 130 .

10. Kenneth Hopkins et al., Estadística Básica para las Ciencias Sociales y del Comportamiento. México: Prentice-Hall Hispanoamericana, S.A. 1997, pág. 89.

\section{Referencias bibliográficas}

Aiken, Lewis. Test psicológicos y evaluación. México: Prentice Hall Hispanoamerica, S.A. 1996.

Ary, Donald, Cheser, Lucy y Razavieh, Asghar. Introducción a la investigación pedagógica. México: McGrawHill. 1998. 
Hopkins, Kenneth D. et al. Estadística Básica para las Ciencias Sociales y del Comportamiento. México: PrenticeHall Hispanoamericana, S.A. 1997.

Kerlinger, Fred. Investigación del comportamiento. México: McGraw-Hill. 1998.

Lanza, Hilda. "La evaluación de los sistemas educativos en Iberoamérica: algunas reflexiones en torno a su especificidad". Madrid: Ponencia en el Congreso Internacional de Evaluación de la Calidad. 1996.

MEP. Informe nacional de resultados de Bachillerato 1998. San José: Mi- nisterio de Educación Pública. 1999.

MEP. Reglamento de Evaluación de los Aprendizajes. San José: Ministerio de Educación Pública. 2000.

Popham, W. James. “Por qué las pruebas estandarizadas no miden la calidad educativa?" http://www.grade.org. pe/gtee-preal, 1999.

Runyon, R. Y Haber, A. Estadística para las ciencias sociales. Estados Unidos: Addison-Wesley Iberoamericana, S.A. 1992.

Tania E. Moreira Mora Asesora Nacional de Macroevaluación de la División de Control de Calidad y Macroevaluación del Sistema Educativo del Ministerio de Educación Pública 